\title{
Asia Pacific
}

\section{Changes in skin reactivity and associated factors in patients sensitized to house dust mites after 1 year of allergen-specific immunotherapy}

Jeong-Yeop Son ${ }^{1}$, Mann-Hong Jung ${ }^{1}$, Kwang-Wook Koh ${ }^{2}$, Eun-Kee Park ${ }^{3}$, Jeong-Hoon Heo ${ }^{4}$, Gil-Soon Choi ${ }^{1}$, and Hee-Kyoo Kim,*

\footnotetext{
'Department of Internal Medicine, Kosin University College of Medicine, Busan 49267, Korea

${ }^{2}$ Department of Preventive Medicine, Kosin University College of Medicine, Busan 49267, Korea

${ }^{3}$ Department of Medical Humanities and Social Medicine, Kosin University College of Medicine, Busan 49267, Korea

${ }^{4}$ Department of Molecular Biology \& Immunology, Kosin University College of Medicine, Busan 49267, Korea
}

Background: Allergen-specific immunotherapy (SIT) can significantly improve symptoms and reduce the need for symptomatic medication.

Objective: The aim of this study was to investigate changes in skin reactivity to house dust mites (HDMs) as an immunologic response and associated factors after 1 year of immunotherapy.

Methods: A total of 80 patients with allergic airway diseases who received subcutaneous SIT with HDMs from 2009 to 2014 were evaluated. The investigated parameters were basic demographic characteristics, skin reactivity and specific IgE for HDM, serum total lgE level, blood eosinophil counts, and medication score.

Results: The mean levels of skin reactivity to HDMs, blood eosinophil counts, and medication scores after 1 year were significantly reduced from baseline. In univariate comparison of the changes in skin reactivity to HDMs, age $\leq 30$ years, HDMs only as target of immunotherapy, and high initial skin reactivity $(\geq 2)$ to HDMs were significantly associated with the reduction in skin test reactivity. In multivariate analysis, high initial skin reactivity and HDMs only as target allergens were significantly associated with changes in skin reactivity to HDMs. In the receiver operating characteristic curve of the initial mean skin reactivity to HDMs for more than $50 \%$ reduction, the optimal cutoff value was 2.14

Conclusion: This study showed significant reductions in allergen skin reactivity to HDMs after 1 year of immunotherapy in patients sensitized to HDMs. The extent of initial allergen skin reactivity and only HDMs as target allergen were important predictive factors for changes in skin reactivity.

Key words: Allergen immunotherapy; Skin reactivity; House dust mites

*Correspondence: Hee-Kyoo Kim

Department of Internal Medicine, Kosin University College of Medicine, 262 Gamcheon-ro, Seo-gu, Busan 49267, Korea

Tel: +82-51-990-6249

Fax: +82-51-990-6249

E-mail: naum67@naver.com

Received: March 31, 2017

Accepted: April 25, 2017
This is an Open Access article distributed under the terms of the Creative Commons Attribution. Non-Commercial License (http://creativecommons. org/licenses/by-nc/4.0/) which permits unrestricted non-commercial use, distribution, and reproduction in any medium, provided the original work is properly cited. 


\section{INTRODUCTION}

Allergen-specific immunotherapy (SIT) is the only method of therapy that targets the sensitized allergens of IgE-mediated allergic diseases and can potentially change the natural progress of such diseases [1-3]. Although allergens against pollens and bee venom have been most widely studied, SIT has also been shown to be effective against allergies to house dust mites (HDMs) [4]. To date, the clinical efficacy of immunotherapy, including improvement of symptoms and reduction of medication, has been verified in patients with asthma, rhinitis, and allergy to bee venom [1-7]. Various immunological mechanisms for the efficacy of SIT have been suggested, including the generation of $\lg G$ antibodies that can block the specific IgE antigen; reduction of the activity of type 2 helper $T$ cells by inducing immunoreactive cytokines of type 1 helper $T$ cells, such as interferon- $\gamma$ and interleukin (IL)-2; and induction of unresponsiveness or tolerance to allergens through the generation of regulatory $T$ cells that generate cytokines such as IL-10 and transforming growth factor- $\beta$ [8-10]. The conventional application method for allergen administration is subcutaneous injection, but the efficacy of sublingual administration has recently been widely acknowledged [11-13]. The type or number of injected antigens may differ according to the degree of sensitization of immunotherapy patients to antigens, leading to variations in the immunoclinical responses of patients [14-18].

Immunotherapy requires at least 3 years of regular injections, and patients experience clinical effects 6 to 7 months after initiation of the therapy [7]. The effects of immunotherapy are divided into short-term effects, which emerge in the first year of treatment; long-term effects that appear in 3 to 5 years; and effects that arise after the suspension of therapy [2]. The degree of improvement of allergic symptoms, together with changes in the level of specific antibodies, cytokines, and airway hypersensitivity, are used as indicators of clinical efficacy during the immunotherapy period $[1,2,7]$. However, the results of relevant studies show little consistency due to differences in the degree of changes according to the type of immunotherapeutic agent, subject, and treatment modalities, as well as differences in study design and analytical methods. The degree of change in skin reactivity to target allergens of immunotherapy is an indicator that is easy to clinically evaluate. Most studies on the efficacy of immunotherapy have demonstrated that skin reactivity was reduced, and suggested that this change could influence the efficacy of the therapy $[3-6,8,19,20]$. Nonetheless, those studies failed to emphasize the clinical significance of changes in skin reactivity, and the factors that affect such changes have not been fully identified. The use of changes in skin reactivity as an index for objective evaluation of the effect of immunotherapy after a certain time period could improve communication with patients regarding the results of the therapy. Furthermore, if the factors associated with such changes are identified, it might be possible to predict the efficacy of immunotherapy before beginning treatment.

This study investigates how the level of skin reactivity to HDM allergens, among other immunoclinical indicators, is affected by 1 year of SIT in patients sensitized to HDMs, with the goal of identifying associated clinical factors.

\section{MATERIALS AND METHODS}

\section{Subjects}

We conducted a retrospective search in the hospital electronic database of patients who received SIT for allergic rhinitis with or without bronchial asthma in the Allergy Clinic at Kosin University Gospel Hospital from January 2009 to August 2014. These patients did not have severe asthma, decreased immune function, malignant tumors, or comorbidity such as cardiovascular diseases, and were eligible for receiving immunotherapy. Asthma was diagnosed when a patient experienced at least one of persistent coughing, wheezing, breathing difficulties, and chest pressure for more than 3 weeks, and showed positivity in either the airway reversibility test or bronchial provocation test. Allergic rhinitis was diagnosed in patients who had repeatedly experienced runny and itchy nose, sneezing, and sinus congestion, regardless of common cold symptoms, had positive response with skin prick test or lgE against specific allergens in their blood.

The study population was patients aged between 5 and 60 with allergic rhinitis or asthma. All patients received sufficient explanation about immunotherapy before beginning treatment and the patients or their legal guardians consented to a survey of their medical records. Immunotherapy for specific allergies, including HDMs, was carried out for 1 year according to planned schedules, and the patients were subjected to skin prick tests before and after the therapy. Patients excluded from the analysis of this study were those who received immunotherapy for allergens other than HDMs, did not follow the regular schedule of 
immunotherapy, did not complete the full course of treatment, or did not have a skin prick test both before and after the therapy.

We received approval for this study from the ethics committee at Kosin University Gospel Hospital.

\section{Methods}

Skin reactivity to Dermatophagoides farina (Df) or Dermatophagoides pteronyssinus (Dp), level of specific lgE (slgE) for HDMs (slgE for HDM), serum total IgE level (normal $\leq 300 \mathrm{IU} / \mathrm{mL}$ ), blood eosinophil counts (normal $\leq 350 / \mu \mathrm{L}$ ), and average daily medication for the previous 2 weeks were surveyed.

The patients' age at onset of immunotherapy, sex, presence of asthma or allergic rhinitis, type of allergen subjected to specific immunotherapy, and method of initial therapy were also included in the analysis.

\section{Skin prick test}

A drop of allergen extract solution (Allergopharma, Reinbek, Germany) including 37 types of major inhaled allergens (including pasture grass, plants and trees, weed pollen, HDMs, mold, and fur of dogs and cats), a positive control solution (histamine), and a negative control solution (normal saline) were applied to the backs or forearms of the test subjects. The skin underneath the drops was pricked with a 26-G needle, allowing the solutions to permeate under the skin. After 15 minutes the wheal response was observed and the average diameter of the wheal was calculated by measuring the length and perpendicular bisector of the longest axis. Subjects were diagnosed as positive when a wheal did not appear for the negative control solution and the average diameter of the wheal formed by the allergen extract solution was both larger than $3 \mathrm{~mm}$ and larger than the wheal formed by the positive control solution. All patients subjected to the test were positive for at least one of the 2 types of HDM allergen. The level of skin reactivity to allergen was expressed as the proportion of the positive control esponse $(A / H)$. Skin reactivity to HDMs, as applied to univariate and multivariate analysis, was calculated using the average value of skin reactivity to Df and Dp.

\section{Blood level of slgEs for HDMs}

The level of slgEs for HDMs in the blood was measured using the immunoCAP system (Phadia AB, Uppsala, Sweden) and the range of the levels was described as $0-100 \mathrm{kU} / \mathrm{L}$.

\section{Allergen-specificimmunotherapy}

For all patients, immunotherapy was administered through subcutaneous injection of Novo-Helisen (Allergopharma). The target allergen for immunotherapy was determined for each patient through a survey of their medical histories and by checking for the presence of specific lgE by the skin prick test. The patients were divided into 2 groups: those who were treated only with HDM allergens, and those who were treated with a mixture of HDMs and other allergens. The immunotherapy schedule involved 2 phases, an initial (build-up) phase and a maintenance period. The initial phase of subcutaneous immunotherapy was initiated with $0.1 \mathrm{~mL}$ of a 1:1,000 dilution of maintenance concentrate, and injection doses were increased weekly to reach a target maintenance dose of concentrate $(0.8 \mathrm{~mL})$. Subsequently, maintenance therapy was carried out once a month

\section{Medication score}

The medication score was calculated as average daily medication (1 point for antihistamine, leukotriene modifier, decongestant, inhaled/intranasal steroids, 2 points for oral corticosteroids) for the previous 2 weeks at both time points of the analysis.

\section{Statistical analysis}

IBM SPSS statistics version 20 (IBM Co., Armonk, NY, USA) was used for statistical analysis. The extent of changes in immunoclinical indicators including skin reactivity level, serum total IgE level, blood eosinophil counts, and daily average medication score after 1 year of immunotherapy was analyzed using paired $t$ test, whereas comparisons of changes in skin reactivity level to nominal or category variables were analyzed using attest. For some parameters, continuous variables were converted into 2 categorical variables. The variable cutoff for age was set at 30 years, the median value of the age of patients eligible for immunotherapy, and the skin reactivity variable was set at reactivity level 2 , which was also the median value. In addition, serum total lgE and blood eosinophil counts were each divided into 2 groups according to normal range. Multiple regression analysis was carried out to determine factors that affected changes in the skin reactivity level with correction for confounding variables. The parameters that showed significant 
effects in the univariate analysis, including age, degree of initial skin response, and type of immunotherapy allergen, were included as independent variables, and the final result was analyzed using stepwise selection. To predict a 50\% reduction in the level of skin reactivity to HDM allergens after 1 year of immunotherapy, receiver operating characteristic $(\mathrm{ROC})$ curve analysis for the level of initial skin reactivity was conducted, and the optimal cutoff value for the maintenance of high sensitivity and specificity was calculated. The calculated value was considered statistically significant when its $p$ value was less than 0.05 .

\section{RESULTS}

\section{Clinical characteristics of patients}

A total of 80 patients were analyzed in this study. The mean age was 22.7 years (range: 5-60 years) and there were more men $(n=51)$ than women. Among the study population, 46 patients had only allergic rhinitis and 34 had both asthma and allergic rhinitis. Only HDMs were used as the target allergen of immunotherapy for $58 \%$ of patients. For patients who received a combination of allergens, most had 2 additional allergens. Tree pollen was the most prevalent additional allergen, in $64.7 \%$ of these patients, followed by weed pollen at $17.6 \%$, grass pollen at $14.7 \%$, dog or cat fur at $11.7 \%$, and mold at 2.9\% (Table 1).

The patients' initial skin reactivity to Dp (European HDMs) and Df (American HDMs) were similar, at 2.2 and 2.3, respectively. Notably, the IgE level was higher for Dp (34.6 kU/L) than for Df (25.2 kU/L). Serum total lgE was $460 \mathrm{JU} / \mathrm{mL}$, and blood eosinophil count was $330.5 / \mu \mathrm{L}$. The ratio of specific IgE to serum total IgE (multiplied by 100) was 14.5 and 9.7 for Dp and Df, respectively. The daily average medication score was 2.8 (Table 2).

Medicines initially administered to the patients included antihistamines (96.4\% of patients), steroid nasal spray (75\%), leukotriene modifiers (85\%), inhaled corticosteroids (11.3\%), inhaled corticosteroids and long-duration bronchodilator (8.8\%), and other medicine such as decongestants (11.3\%).

\section{Changes in immunoclinical indicators after 1 year of immunotherapy}

The skin reactivity of the patients was checked an average of 12 months after the start of immunotherapy. The degree of changes in skin reactivity to HDMs was statistically significant for both Dp and Df. The skin reactivity level decreased from 2.2 to 1.2
Table 1. Baseline characteristics of subjects $(n=80)$

\begin{tabular}{lc}
\hline Characteristic & Value \\
\hline Age (yr), mean (range) & $22(5-60)$ \\
\hline Sex, male:female & $51(63.7): 29(36.2)$ \\
\hline Underlying airway diseases & \\
\hline Allergic rhinitis only & $46(57.5)$ \\
\hline Bronchial asthma with allergic rhinitis & $34(42.5)$ \\
\hline Target allergens for immunotherapy & \\
\hline HDM only & $46(57.5)$ \\
\hline HDM mixed with other allergens & $34(42.5)$ \\
\hline 1 Allergen & $9 / 34(26.4)$ \\
\hline 2 Allergens & $14 / 34(41.2)$ \\
\hline 3 Allergens & $6 / 34(17.6)$ \\
\hline 4 Allergens & $4 / 34(11.8)$ \\
\hline 5 Allergens & $1 / 34(2.9)$ \\
\hline
\end{tabular}

Values are presented as number (\%) unless otherwise indicated. HDM, house dust mite.

Table 2.Baseline immunoclinical characteristics of subjects

\begin{tabular}{lc}
\hline Characteristic & Mean (range) \\
\hline Initial allergen reactivities for HDM & \\
Dp & $2.2(1-6.0)$ \\
Df & $2.3(1-6.5)$ \\
Specific serum lgE levels (kU/L) for HDM & \\
\hline Dp & $34.6(10-00)$ \\
Df & $25.2(10-100)$ \\
Serum total lgE levels (IU/mL) & $460(6.2-5,000)$ \\
Serum slgE/total IgE ratios $(\times 100)$ for HDM & \\
\hline Dp & $14.5(0.1-67.4)$ \\
Df & $9.7(0.1-47.9)$ \\
Blood eosinophil (counts/ $\mu \mathrm{L})$ & $330.5(10-1,190)$ \\
Medication score & $2.8(1-5)$ \\
\hline
\end{tabular}

HDM, house dust mites; Dp, Dermatophagoides pteronyssinus; Df, Dermatophagoides farina.

for Dp and from 2.3 to 1.3 for Df ( $p<0.001$ for each). The HDMspecific lgE level in the blood increased slightly from the initial level, to $39.3 \mathrm{kU} / \mathrm{L}$ and $33.7 \mathrm{kU} / \mathrm{L}$ for Dp and Df respectively, but the increases were not statistically significant $(p=0.56$ and $p=$ 0.55 , respectively).

Serum total IgE levels increased slightly to $613.7 \mathrm{IU} / \mathrm{mL}$, but the difference was not statistically significant $(p=0.36$ ). The ratio of specific lgE to total serum lgE (specific lgE/total $\lg E \times 100$ ) 


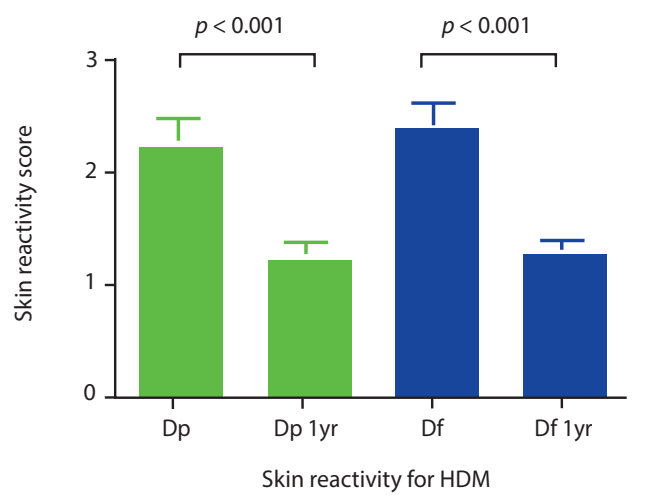

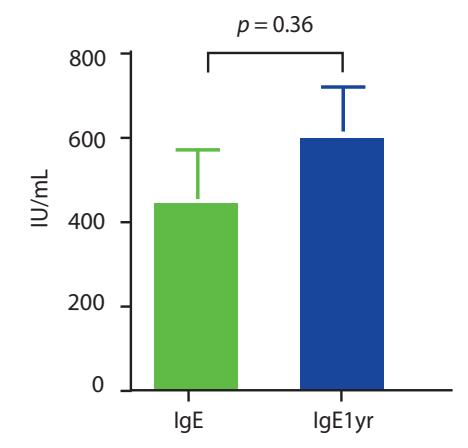

C

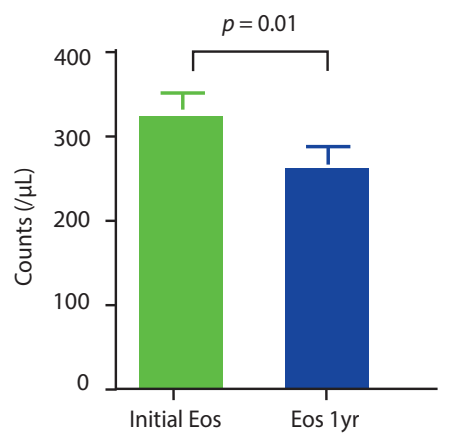

E

A

Blood Eosinophil

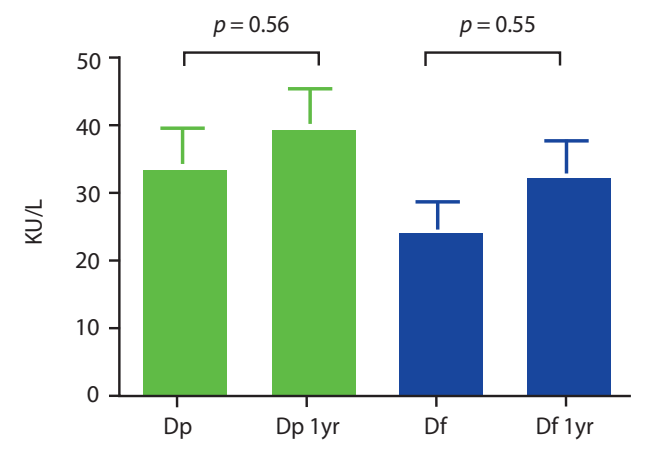

B

Specific lgE for HDM
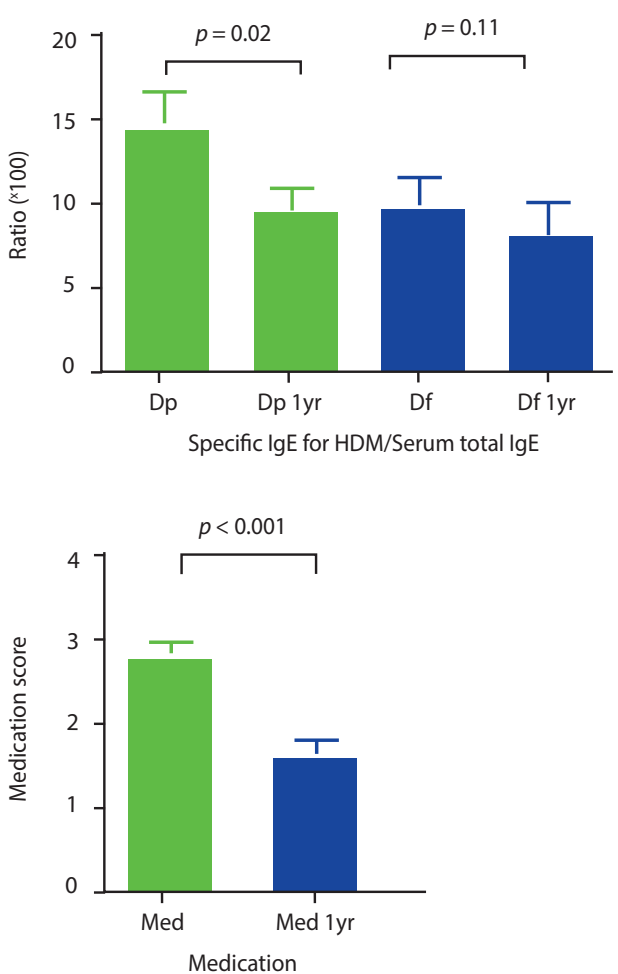

Fig. 1. Changes of skin reactivity (A), serum specific lgE level (B), serum total $\lg E(C)$, serum specific $\lg E /$ total $\lg E(X 100)(D)$, blood eosinophils $(E)$, and medication score (mean number/day) (F) between baseline and after 1 year of therapy. HDM, house dust mites; Dp, Dermatophagoides pteronyssinus; Df, Dermatophagoides farinae.

decreased by an average of 9.7 for Dp ( $p=0.03$ ) and 8.3 for Df ( $p$ $=0.11$ ), although the degree of change differed according to the type of allergen. Blood eosinophil counts showed a significant decrease to $265.9 / \mu \mathrm{L}(p=0.01$ ) and the daily average number of administered medicines also decreased significantly to $1.6 /$ day ( $p<$ 0.001) (Fig. 1).

\section{Univariate analysis of change in skin reactivity to HDMs after 1 year of immunotherapy}

The level of skin reactivity decreased by 1.34 for the $\leq 30$ age group, which was a significantly greater decrease than that for the $>30$ age group $(0.69, p=0.04)$. There was no significant difference according to gender or disease targeted by the immunotherapy. The level of reactivity decreased by an average 
Table 3. Skin reactivity changes for house dust mites after 1 year according to clinical parameters

\begin{tabular}{|c|c|c|c|}
\hline Characteristic & Number & Change, mean \pm SEM & $p$ value \\
\hline Age group (yr) & & & 0.04 \\
\hline$\leq 30$ & 61 & $1.34 \pm 0.19$ & \\
\hline$>30$ & 19 & $0.69 \pm 0.43$ & \\
\hline Sex & & & 0.27 \\
\hline Male & 51 & $0.97 \pm 0.22$ & \\
\hline Female & 29 & $1.20 \pm 0.22$ & \\
\hline Underlying airway diseases & & & 0.96 \\
\hline Allergic rhinitis only & 46 & $0.98 \pm 0.22$ & \\
\hline Bronchial asthma with allergic rhinitis & 34 & $1.15 \pm 0.25$ & \\
\hline Target allergens for immunotherapy & & & 0.03 \\
\hline HDM only & 46 & $1.72 \pm 0.26$ & \\
\hline HDM with other allergen(s) & 34 & $0.86 \pm 1.29$ & \\
\hline Initial mean allergen reactivities for HDM & & & $<0.001$ \\
\hline Allergen reactivity $<2$ & 44 & $0.12 \pm 0.07$ & \\
\hline Allergen reactivity $\geq 2$ & 36 & $2.18 \pm 0.25$ & \\
\hline \multicolumn{4}{|l|}{ Initial serum total lgE levels (IU/mL) } \\
\hline $0-300$ & 31 & $1.33 \pm 0.28$ & 0.33 \\
\hline$>300$ & 21 & $0.43 \pm 0.31$ & \\
\hline Blood eosinophil counts $(/ \mu \mathrm{L})$ & & & 0.47 \\
\hline$\leq 350$ & 30 & $1.32 \pm 0.29$ & \\
\hline$>350$ & 45 & $1.07 \pm 0.19$ & \\
\hline
\end{tabular}

SEM, standard error of the mean; HDM, house dust mites.

Table 4. Effects of immunoclinical factors on skin reactivity changes for house dust mites by multiple regression analysis

\begin{tabular}{lcccc}
\hline Variable & Beta & SE & $95 \% \mathrm{Cl}$ & \multicolumn{1}{c}{$p$ value } \\
\hline HDM only as target & 0.543 & 0.234 & $0.78-1.01$ & $1.52-2.50$ \\
Initial skin reactivity, $\geq 2$ & 2.006 & 0.246 & $-0.51-0.63$ & 0.001 \\
Age, $\leq 30$ years & 0.058 & 0.286 & -973 \\
\hline
\end{tabular}

Beta, estimated regression coefficient, SE,standard error; Cl, confidence interval.

Adjusted $R^{2}=0.508$.

of 1.72 for the group of patients who received immunotherapy against HDMs only, a significant difference from the decrease by 0.12 for patients treated for HDM and additional allergens. When the initial skin reactivity level was 2 or higher, the skin reactivity level decreased by 2.18 on average, significantly greater than the decrease by 0.12 observed for patients whose initial level was lower than $2(p<0.001)$. There was also a significant difference in the ratio of decrease in skin reactivity from the initial level (change in skin reactivity/initial skin reactivity $(\times 100)$ between the
2 groups ( $p<0.001$ ). No significant difference in skin reactivity was observed for different levels of initial serum total IgE (higher or lower than $300 \mathrm{IU} / \mathrm{mL}, p=0.33$ ) or blood eosinophil counts (higher or lower than 350/uL, $p=0.47$ ) (Table 3).

\section{Multivariate analysis of change in skin reactivity to HDMs after 1 year of immunotherapy}

For multivariate analysis of the factors affecting changes in the level of skin reactivity after 1 year of immunotherapy, 


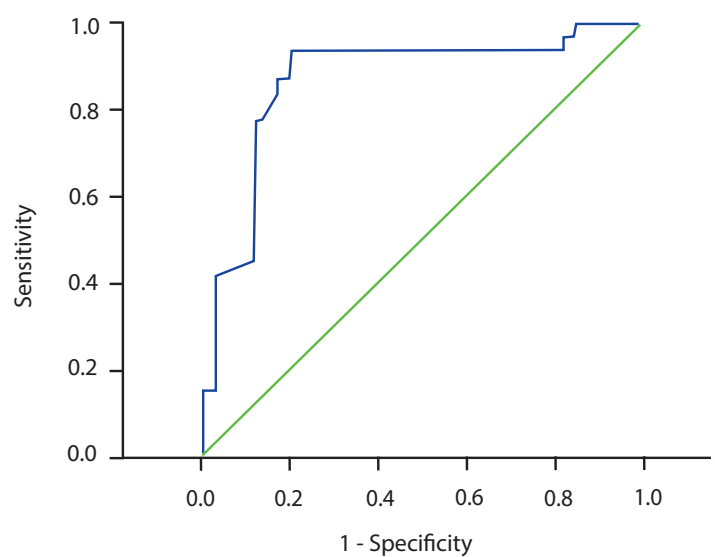

Fig. 2. Receiver operating characteristic curves of initial mean skin reactivity to house dust mites for more than $50 \%$ reduction of skin reactivity. The area under the curve was 0.87 ( $95 \%$ confidence interval, $0.776-0.965, p<0.001)$. With a cutoff value of 2.14 , the sensitivity and specificity were 0.90 and 0.80 , respectively.

multiple regression analysis was carried out for the parameters of age, initial skin reactivity level, and target allergens used for immunotherapy, factors that showed statistically significant differences in the univariate analysis. The modeling designed for the analysis was proven to be statistically significant, as its significance probability was lower than 0.05 by F-test. The corrected determination efficient $R^{2}$ at 0.508 demonstrated substantial explanation ability. Among the analyzed parameters, initial skin reactivity level showed the most significant influence $(p<0.001)$, followed by the use of HDM-only versus multiple allergen therapies $(p=0.023)$. There was no significant difference between patients aged under or over $30(p=0.97)$ (Table 4).

\section{ROC curve analysis of initial skin reactivity for the prediction of $50 \%$ reduction of skin reactivity to HDMs after $\mathbf{1}$ year of immunotherapy}

ROC curve analysis was conducted on the initial skin reactivity level in order to predict a reduction of the level of skin reactivity to HDMs greater than $50 \%$ after 1 year of immunotherapy. The area under curve was 0.87 with statistical significance $(p<0.001)$, and the optimal value was 2.14. At the levels of the optimal value, the sensitivity and specificity were 0.90 and 0.80 , respectively (Fig. 2).

\section{DISCUSSION}

The majority of HDMs found in nature belong to 2 species,
Dermatophagoides farinae and Dermatophagoides pteronyssinus, which show a high level of cross-reactivity [21, 22]. In Korea, $40 \%-60 \%$ of patients with respiratory allergy and approximately $30 \%$ with atopic dermatitis are sensitized to HDMs. Children and teenagers sensitized to HDMs show a higher incidence of asthma and allergic rhinitis [23-25]. The HDM allergens account for a high proportion of the allergens used for immunotherapy in Korea, and can therefore be regarded as the most common allergens in the country [26].

Previous studies have suggested variation in the time for improvement of allergic symptoms in patients treated with immunotherapy. This period can be affected by a variety of factors, including the method of allergen injection, type and amount of vaccine, and severity of symptoms, but in most cases, the effect of immunotherapy usually begins around 7 to 8 months after starting subcutaneous therapy $[7,8]$. Most of the subjects in this study actually demonstrated improvement of symptoms, but they did not complete standardized checklists as surveys of subjective symptoms during immunotherapy. However, because objective immunoclinical variables, including the level of skin reactivity, demonstrated significant changes, the immunotherapy administered in this study can be considered effective.

The level of skin reactivity, allergen-specific lgE, lgG1, and IgG4 in the blood, and total serum IgE have been suggested as indicators of the immunological changes that occur during immunotherapy $[1-3,10,19,20,26]$. The skin reactivity and specific lgE levels reflect the extent of sensitivity of the patient to allergens, and specific lgG is known to act as a blocking antibody for lgE. The level of skin reactivity tends to remain constant for 6 months after beginning immunotherapy and decreases gradually after 1 year; however, there have been reports of decreased skin reactivity at earlier time points $[20,26]$. Immunotherapy with pollen as the target allergen has shown similar results $[19,26]$. Although the level of skin reactivity decreased in this study, the amount of specific lgE antibody against HDMs increased. This is consistent with many other previous reports; however, decreased IgE levels have also been reported, even within a year after the beginning of therapy $[6,20,26,27-29]$. The mechanism behind the decrease in skin reactivity earlier than the decrease in specific antibody level in the blood has not yet been identified, but it is probable that changes in the activation of allergy effectors that affect skin reactivity, including mast cells and basophils, are 
induced earlier than changes in antibody generation by plasma cells $[10,20]$.

Increased levels of IgG1 and IgG4 are significantly correlated with the blocking of $\mathrm{lgE}$ antibodies and clinical improvement of airway hypersensitivity $[2,8,26,30]$. IgG1 and $\lg G 4$ concentrations are known to increase during early stages of immunotherapy, and can be used as markers for the evaluation of therapeutic efficacy, This study did not investigate specific lgG subtype levels in the subjects because there is currently no commercial test available.

The level of serum total IgE tended to increase 1 year after immunotherapy onset, consistent with previous reports $[6,20,26]$. Additional studies have focused on changes in the slgE/total lgE ratio and report that high initial slgE/total lgE ratio is associated with the prognosis of the efficacy of immunotherapy [31]. In this study, the slgE/total IgE ratio for Dp showed a significant decrease from the level before therapy onset but the ratio for Df did not. However, in a previous study of immunotherapy for 3 years, the ratio for that increased the from initial level [32]. Further research is needed to determine that clinical meaning of such changes1 year after the beginning of immunotherapy [32].

Eosinophils are major effector cells for allergic diseases that, when activated, secrete chemical mediators such as eosinophilic cationic protein. The influx of eosinophils to the target organ is reduced during immunotherapy, potentially affecting the latephase response to the allergen $[1,8,10]$. In addition, a change in eosinophilic chemical mediators at the target site is suggested to be an indicator of the efficacy of immunotherapy $[2,30]$. A change in eosinophils in peripheral blood 1 year after the beginning of immunotherapy is not consistently reported, but one Korean study demonstrated a significant decrease in blood eosinophils with subcutaneous immunotherapy [20], similar to the results of this study. There was no difference in the degree of skin reactivity according to whether blood eosinophil counts were within or outside of the normal range before the beginning of therapy.

In this study, we focused on changes in skin reactivity level as an indicator of the efficacy of immunotherapy because it is a convenient, fast, inexpensive test for immunoclinical changes. Importantly, skin reactivity level is considered a reliable indicator for the evaluation of immunotherapy efficacy, and most relevant studies have demonstrated decreases in skin reactivity after immunotherapy $[8,19,20,29]$. Additional studies have shown that a decreased skin reactivity level is associated with decreases in the number of skin mast cells and allergic symptoms [20, 33, 34].
Therefore, identifying the factors associated with skin reactivity may provide some predictive insights during the initial phase. For this study, initial skin reactivity level was the most significant factor affecting the absolute and ratio decrease in skin reactivity level after 1 year, suggesting that a higher initial skin reactivity level results in greater efficacy of immunotherapy.

Age group did not show a significant influence on changes in skin reactivity level in multivariate regression analysis, unlike in univariate analysis. This seems to be because the level of initial skin reactivity was higher for patients under 30 than for older patients. Despite some opinions that immunotherapy is more effective in younger patients, differences in the changes in skin reactivity level for different age groups have not been identified [35]. Therefore, additional large-scale studies are required to analyze the efficacy of immunotherapy indifferent age groups.

Patients who received HDM-only immunotherapy displayed a statistically significant decrease in skin reactivity level compared with patients who received immunotherapy against multiple antigens, indicating that the lower the number of target allergens, the better the efficacy of immunotherapy. To date, few studies have directly compared the efficacy of single- and multiallergen immunotherapy. However, it has been reported that immunotherapy with a single allergen is more effective than that with multiple allergens with regard to immunological changes, including decrease in skin reactivity level [16-18]. However, as there are some reports of the clinical efficacy of multiple-allergen immunotherapy, further research will have to be conducted [15-17, 36].

There were some limitations to this study. First, the analysis was conducted only in patients who received immunotherapy; therefore, comparison against a control group was impossible. Second, in this retrospective study the patient medical records were insufficient for analysis of standardized information about changes in subjective symptoms perceived by patients. Third, the limited number of subjects did not allow more detailed subgroup analyses.

In summary, patients sensitized to HDMs who received immunotherapy showed a significant decrease in skin reactivity level to HDM allergens1 year after the beginning of therapy. Major factors that affected this change were the level of initial skin reactivity and the inclusion of only HDM allergens in the therapy. Further studies are needed to analyze changes in skin reactivity level and target site sensitivity to allergens after longterm immunotherapy on a larger scale. 


\section{REFERENCES}

1. Cox L, Esch RE, Corbett M, Hankin C, Nelson M, Plunkett G. Allergen immunotherapy practice in the United States: guidelines, measures, and outcomes. Ann Allergy Asthma Immunol 2011;107:289-99.

2. Cox L, Nelson H, Lockey R, Calabria C, Chacko T, Finegold I, Nelson M, Weber R, Bernstein DI, Blessing-Moore J, Khan DA, Lang DM, Nicklas RA, Oppenheimer J, Portnoy JM, Randolph C, Schuller DE, Spector SL, Tilles S, Wallace D. Allergen immunotherapy: a practice parameter third update. J Allergy Clin Immunol 2011;127(1 Suppl):S1-55.

3. Jacobsen L, Wahn U, Bilo MB. Allergen-specific immunotherapy provides immediate, long-term and preventive clinical effects in children and adults: the effects of immunotherapy can be categorised by level of benefit -the centenary of allergen specific subcutaneous immunotherapy. Clin Transl Allergy 2012;2:8.

4. Calderon MA, Casale TB, Nelson HS, Demoly P. An evidence-based analysis of house dust mite allergen immunotherapy: a call for more rigorous clinical studies. J Allergy Clin Immunol 2013;132:1322-36.

5. Nanda A, O'connor M, Anand M, Dreskin SC, Zhang L, Hines B, Lane D, Wheat W, Routes JM, Sawyer R, Rosenwasser LJ, Nelson HS. Dose dependence and time course of the immunologic response to administration of standardized cat allergen extract. J Allergy Clin Immunol 2004;114:1339-44.

6. Yukselen A, Kendirli SG, Yilmaz M, Altintas DU, Karakoc GB. Effect of one-year subcutaneous and sublingual immunotherapy on clinical and laboratory parameters in children with rhinitis and asthma: a randomized, placebo-controlled, double-blind, double-dummy study. Int Arch Allergy Immunol 2012;157:288-98.

7. Jutel M, Agache I, Bonini S, Burks AW, Calderon M, Canonica W, Cox L, Demoly P, Frew AJ, O'Hehir R, Kleine-Tebbe J, Muraro A, Lack G, Larenas D, Levin M, Nelson H, Pawankar R, Pfaar O, van Ree R, Sampson H, Santos AF, Du Toit G, Werfel T, Gerth van Wijk R, Zhang $L$, Akdis CA. International consensus on allergy immunotherapy. J Allergy Clin Immunol 2015;136:556-68.

8. Akdis M, Akdis CA. Mechanisms of allergen-specific immunotherapy. J Allergy Clin Immunol 2007;119:780-91.

9. Larché M, Akdis CA, Valenta R. Immunological mechanisms of allergen-specific immunotherapy. Nat Rev Immunol 2006;6:761-71.

10. Jutel M, Agache I, Bonini S, Burks AW, Calderon M, Canonica W, Cox L, Demoly P, Frew AJ, O'Hehir R, Kleine-Tebbe J, Muraro A, Lack G, Larenas D, Levin M, Martin BL, Nelson H, Pawankar R, Pfaar O, van Ree R, Sampson H, Sublett JL, Sugita K, Du Toit G, Werfel T, Gerth van Wijk R, Zhang L, Akdis M, Akdis CA. International Consensus on Allergen Immunotherapy II: Mechanisms, standardization, and pharmacoeconomics. J Allergy Clin Immunol 2016;137:358-68.

11. Tonnel AB, Scherpereel A, Douay B, Mellin B, Leprince D, Goldstein $N$, Delecluse $P$, Andre C. Allergic rhinitis due to house dust mites: evaluation of the efficacy of specific sublingual immunotherapy. Allergy 2004;59:491-7.

12. Cingi C, Bayar Muluk N, Ulusoy S, Acar M, Şirin S, Çobanoğlu B, Birdane L, Kalaycık Ç, Çakır BÖ, Oğhan F, Aynacı S, Erdoğmuş N, Yıldırım Ö, Şahin E, Bulut F, Aksoy MA, Türe N, Bal C. Efficacy of sublingual immunotherapy for house dust mite allergic rhinitis. Eur Arch Otorhinolaryngol 2015;272:3341-6.

13. Kim ST. Outcome of sublingual immunotherapy in patients with allergic rhinitis sensitive to house dust mites. Allergy Asthma Immunol Res 2015;7:99-100.

14. Kim SH, Shin SY, Lee KH, Kim SW, Cho JS. Long-term effects of specific allergen immunotherapy against house dust mites in polysensitized patients with allergic rhinitis. Allergy Asthma Immunol Res 2014;6:535-40.

15. Soyyigit S, Guloglu D, Ikinciogullari A, Secil D, Oztuna D, Mungan $D$, Misirligil Z, Sin BA. Immunologic alterations and efficacy of subcutaneous immunotherapy with Dermatophagoides pteronyssinus in monosensitized and polysensitized patients. Ann Allergy Asthma Immunol 2016;116:244-51.e2.

16. Kim KW, Kim EA, Kwon BC, Kim ES, Song TW, Sohn MH, Kim KE. Comparison of allergic indices in monosensitized and polysensitized patients with childhood asthma. J Korean Med Sci 2006;21:1012-6.

17. Calderón MA, Cox L, Casale TB, Moingeon P, Demoly P. Multipleallergen and single-allergen immunotherapy strategies in polysensitized patients: looking at the published evidence. J Allergy Clin Immunol 2012;129:929-34.

18. Nelson HS. Specific immunotherapy with allergen mixes: what is the evidence? Curr Opin Allergy Clin Immunol 2009;9:549-53.

19. Panizo C, Cimarra M, González-Mancebo E, Vega A, Senent C, Martín S. In vivo and in vitro immunological changes induced by a short course of grass allergy immunotherapy tablets. J Investig Allergol Clin Immunol 2010;20:454-62.

20. Lee E, Kim MJ, Yang SI, Yu J, Hong SJ. Comparison of short-term effects between subcutaneous and sublingual immunotherapies in children with house dust mite-sensitized allergic rhinitis and asthma. Allergy Asthma Respir Dis. 2015;3:180-6.

21. Kim JH, Jang AS, Jeong SO, Ji YS, Seo HJ, Nam JH, Moon JJ, Baek AR, Park JS, Lee JH, Park SW, Kim DJ, Park CS. The differences of clinical profiles by house dust mite sensitization in patients with asthmatics 
in Soonchunhyang University Hospital cohort. Allergy Asthma Respir Dis 2013;1:50-4

22. Jeong KY, Park JW, Hong CS. House Dust Mite Allergy in Korea: The Most Important Inhalant Allergen in Current and Future. Allergy Asthma Immunol Res 2012;4:313-25.

23. Park HJ, Lim HS, Park KH, Lee JH, Park JW, Hong CS. Changes in allergen sensitization over the last 30 years in korea respiratory allergic patients: a single-center. Allergy Asthma Immunol Res 2014;6:434-43.

24. Lee JE, Ahn JC, Han DH, Kim DY, Kim JW, Cho SH, Park HW, Rhee CS. Variability of offending allergens of allergic rhinitis according to age: optimization of skin prick test allergens. Allergy Asthma Immunol Res 2014;6:47-54.

25. Hur GY, Kim TB, Han MY, Nahm DH, Park JW; Allergen and Immunotherapy Work Group of the Korean Academy of Asthma, Allergy and Clinical Immunology (KAAACI). A survey of the prescription patterns of allergen immunotherapy in Korea. Allergy Asthma Immunol Res 2013;5:277-82.

26. Kim BS, Lee SK, Park HS, Hong SJ. The early changes of humoral immune response after rush immunotherapy with Dermatophagoides farinae (D.f) and Dermatophagoides pteronyssinus (D.p) in house dust mite sensitive asthmatic children. J Asthma Allergy Clin Immunol 2001;21:543-51.

27. Cevit O, Kendirli SG, Yilmaz M, Altintas DU, Karakoc GB. Specific allergen immunotherapy: effect on immunologic markers and clinical parameters in asthmatic children. J Investig Allergol Clin Immunol 2007;17:286-91.

28. Aasbjerg K, Backer V, Lund G, Holm J, Nielsen NC, Holse M, Wagtmann VR, Würtzen PA. Immunological comparison of allergen immunotherapy tablet treatment and subcutaneous immunotherapy against grass allergy. Clin Exp Allergy 2014;44:417-28.

29. Eifan AO, Akkoc T, Yildiz A, Keles S, Ozdemir C, Bahceciler NN, Barlan IB. Clinical efficacy and immunological mechanisms of sublingual and subcutaneous immunotherapy in asthmatic/rhinitis children sensitized to house dust mite: an open randomized controlled trial. Clin Exp Allergy 2010;40:922-32.

30. Kim HB, Lee SY, Kim JH, Kim BS, Seo HJ, Hong SJ. A Three-year Follow-up of D.f- and D.p-Specific Conventional Immunotherapy in Asthmatic Children. Pediatr Allergy Respir Dis 2005;15:26-34.

31. Li Q, Li M, Yue W, Zhou J, Li R, Lin J, Li Y. Predictive factors for clinical response to allergy immunotherapy in children with asthma and rhinitis. Int Arch Allergy Immunol 2014;164:210-7.

32. Peng $H$, Li CW, Lin ZB, Li TY. Long-term efficacy of specific immunotherapy on house dust mite-induced allergic rhinitis in China. Otolaryngol Head Neck Surg 2013;149:40-6.

33. Löfkvist T, Agrell B, Dreborg S, Svensson G. Effects of immunotherapy with a purified standardized allergen preparation of Dermatophagoides farinae in adults with perennial allergic rhinoconjunctivitis. Allergy 1994:49:100-7.

34. Karakoc-Aydiner E, Eifan AO, Baris S, Gunay E, Akturk E, Akkoc T, Bahceciler NN, Barlan IB. Long-term effect of sublingual and subcutaneous immunotherapy in dust mite-allergic children with asthma/rhinitis: a 3-year prospective randomized controlled trial. J Investig Allergol Clin Immunol 2015;25:334-42.

35. Pajno GB, Barberio G, De Luca F, Morabito L, Parmiani S. Prevention of new sensitizations in asthmatic children monosensitized to house dust mite by specific immunotherapy. A six-year follow-up study. Clin Exp Allergy 2001;31:1392-7.

36. Li L, Guan K. Effect on quality of life of the mixed house dust mite/ weed pollen extract immunotherapy. Asia Pac Allergy 2016;6:168-73. 